\title{
A Study of Port Site Infection Following Laparoscopic Cholecystectomy In a teaching Hospital.
}

\author{
Dr. Narayan Das, M.B.B.S, $\mathrm{Ms}^{1}$ Dr Anarsh Debbarma, M.B.B.S, $\mathrm{Ms}^{2}$ Dr. Anup \\ Kumar Saha, M.B.B.S, $\mathrm{Ms}^{3}$ \\ ${ }^{1}$ Assistant Professor, Department Of Surgery \\ Agartala Government Medical College And GBP Hospital, Agartala, West Tripura, 799006. \\ ${ }^{2}$ Registrar, Department Of Surgery, \\ Agartala Government Medical College And GBP Hospital, Agartala, West Tripura, 799006. \\ ${ }^{3}$ Professor And HOD, Department Of Surgery, \\ Agartala Government Medical College And GBP Hospital, Agartala, West Tripura, 799006.
}

\begin{abstract}
:
Background/Aims: To assess the port site infections in patients who underwent Laparoscopic Cholecystectomy in the department of Surgery

at AGMC and GBP hospital and how to prevent port site infection.

Design: Prospective Study

Materials and Methods: In this study, a total number of 100 patients who underwent Laparoscopic Cholecystectomy for Chronic Calcular Cholecystitis from January 2015 to June 2016 at our hospital were observed for port site infections. Culture Sensitivity were taken for all patients who developed Port site infections. Variables like Port site, Port size, causative organisms, Surgery-infection interval were analysed.

Results: The incidence of port site infection in our study is 7\%. The port site infection is more common at Epigastric port site and in $10 \mathrm{~mm}$ port size. Most common organisms are staphylococcus aureus followed by Pseudomonas aeruginosa and atypical mycobacterial infection.

Conclusion: Port site infections in Laparoscopic Cholecystectomy are minor complication, but still these complications are preventable by proper sterilization of laparoscopic instruments.
\end{abstract}

Keyword : Laparoscopic Cholecystectomy, Port site infection(PSI), Prevention.

\section{Introduction}

Laparoscopic Surgery(LS), also termed minimal access surgery, has brought a paradigm shift in the approach to modern surgical Care. Early postoperative recovery, less pain, improved aesthesis and early return to work has led to its popularity both amongst surgeons and patients. Port site infections (PSI) although infrequent,is one of the bothersome complication which undermine the benefits of minimal invasive surgery. Not only does it add to the morbidity of the patient but also spoils the reputation of the surgeon.Despite the advances in the field of antimicrobial agents , Sterilization techniques, surgical techniques, operative room ventilation, port site infections still prevail (1).

The total complication rate of Laparoscopic surgeries was 3.6/1000 procedures and the rate of major complications was $1.4 / 1000$ procedures(2). Recent study concluded that the currently prevalent practice of immersing laparoscopic instruments for 20 minutes in $2 \%$ alkaline glutaraldehyde should be reexamined(3). They also recommend that disinfectant solution used for sterilization was responsible for port site infections. Aim of our study is to assess the port site infections following Laparoscopic Cholecystectomy and its prevention. To prevent infection, proper sterilization and storage of instruments is recommended. According to the definition developed by the united states centre for disease control(CDC), surgical site infections(SSI) were categorized ;(1)Superficial SSI, which involve skin and subcutaneous tissue;(2)Deep SSIs, which involve fascia and muscle layers and (3)Organ/Space SSIs(4). The use of disposable laparoscopic instruments is the Gold standard for prevention of port site infections. Thus proper sterilization of the Laparoscope and instruments is of utmost importance in preventing infectious complications(5).

\section{Material And Methods}

The study included 100 patients who underwent Laparoscopic Cholecystectomy for chronic Calcular Cholecystitis over a period of 18 months from January 2015 to June 2016 in the department of general surgery at Agartala government medical college and GBP hospital, Agartala. Those patients who were converted to open procedures were excluded from the study. In all the patients preoperative preparation was done by complete bath prior to surgery using antiseptic soap and the ports were prepared by shaving method. All patients received 
prophylactic antibiotics during induction of general anaesthesia. All surgeries were done general anaesthesia. Pneumoperitoneum created using veress needle in infraumbilical incision.Through the same incision, a 10mm safety Trocar(primary Trocar) introduced in to the abdominal cavity. All the specimens of gallbladder were extracted through Epigastric port without endobag.All the ports were irrigated with povidone- iodine and normal saline and all $10 \mathrm{~mm}$ port closure was done by hand sewn intermittent suture with 2-0 vicryl. All Laparoscopic instruments were sterilized by $2 \%$ glutaraldehyde solution with a contact time of 20 minutes.Before surgery all the instruments were washed with warm saline. In our study 7 patients (7\%) out of 100 patients developed port site infections(PSI), and all of them were superficial port site infection (SPI).Culture sensitivity were taken from all of the PSI and sent to microbiology department of AGMC and GBP Hospital for analysis.

\section{Results}

In our study, 100 patients were operated including 30 males and 70 females. The incidence of port site infection is $7 \%$ and common in 30-40 years of age group and more common in females. Amongst the port site Epigastric port $(85.6 \%)$ was affected more than umbilical port(14.4\%).Port site infection more commonly seen in $10 \mathrm{~mm}$ epigastric port ( port size). The time interval between surgical period and port site infection very commonly seen after 2 weeks. All port site infection were superficial port site infections. More common causative organisms were staphylococcus aureus followed by pseudomonas aeruginosa and atypical mycobacterial infection. All port site infection were treated with empirical antibiotics and local care of the wound and once getting wound culture sensitivity result, patient was changed to concerned antibiotics and patient with atypical mycobacterial infection was given a full course of ATT.

\section{DISCUSSION}

Laparoscopic surgery is the gold standard for many surgical diseases. Even many patients demand laparoscopic surgery because of the advantages like small incision, less pain and shortened recovery time as well as resulting less post operative scarring(6). All laparoscopic surgeries should be done by the experienced surgeons to avoid major complications

In seith A et al studies (7) port site infection were reported after laparoscopic Cholecystectomy and it was thought to be associated with dropped stone or at the site of physical injury following laparoscopic Cholecystectomy. Amongst the port site Epigastric port (88.2) was affected more than umbilical port $(11.2 \%)$.Wasim Memon et al reported that the causes of port site infections were gross spillage of infected bile ,obesity and umbilical stitch sinus(9).

Mir M A et al studied PSI after elective laparoscopic Cholecystectomy,Incidence was $6.7 \%$ and the cause of PSI could be due to reusable trocars (10).

Port site infection in our study is $7 \%$; similar type results were obtained in other studies like Shindholimeth VV et al $6.3 \%$ (11).

Ten Commandments for preventing PSI (12);

(1) Use of disposable trocars and instruments and adequate availability of properly sterilized reusable trocars to cover all the surgical procedures in a day.

(2) Use of autoclavable laparoscopic hand instruments ;

(3) Use of instruments with good ergonomics, limited joints and facility for proper cleaning of the debris collected in its crevices;

(4) A proper cleaning of the instruments is best achieved by ultrasonic technology. use of autoclaved water for cleaning the instruments after dismantling;

(5) Proper guidelines should be followed regarding the concentration, contact time and cycles of use for instrument sterilization with liquid sterilizing agents;

(6) Use of plasma sterilizer or ethylene oxide in between the consecutive surgery for instrument sterilization.

(7) Avoiding inter-departmental sharing of instruments,such as using instruments for gynaecological or urological procedures.

(8) Avoiding spillage of bile or gut content in the operative area or the port site;

(9) Use of non- porous specimen retrieval bags for retrieving the specimen; and

(10) Through irrigation and cleaning of the of the port site before closure .

\section{Conclusion}

PSI is one of the complication of laparoscopic surgery. The present study is an attempt to make surgeons aware about the complications which occur due to improper sterilization of laparoscopic instruments ending into increased morbidity of patients. The only surgeon who does not encounter complications is one who is not operating. Complication can occur even in the best of hands and it is vital that these are recognized properly and immediately addressed. 


\section{References}

[1]. Prakash K S, Mishra S T, Rathi S ,Meher S ,Mahapatra D. Port site infection in laparoscopic surgery. A review of its Management. World J clin Cases.2015;3(10):864-71.

[2]. Hakki-Sirren, Kurk. A nationwide analysis of laparoscopic complication .Obstet Gynecol.1997;89:108-12.

[3]. Ramesh H ,Prakash K ,Lekhe V, Jacob G, Venugopal A, Venugopal B .Port site tuberculosis after Laparoscopy. Report of eight cases. Surg Endosc.2003;17(6):930-32.

[4]. Garner JS, Jarris JR,Emoris TG,Horan T C,Haques JM.CDC definition for nosocomial infections. Am j infect Control.1998;85:81827.

[5]. Chaudhuri S,Sarkar D ,Mukherji R .Diagnosis and management of atypical mycobacterial infection after laparoscopic Surgery. India J Surg.2010;72(6):438-42.

[6]. Kumar SS,Babu KD, Gree RD,Anpian JC. A study of port site infections in laparoscopic surgeries. IOSR-JDMS.2015;14(4);20-22.

[7]. Seith A, Srivastava DN, Pande GK. Tubercular abdominal abscess following laparoscopic Cholecystectomy. Case report. Trop Gastroenterol.2001;22:216-18.

[8]. Jan AW, Ali IS, Shah AN, Ghani A, Khan M, Khan AS. The frequency of port site infection following Laparoscopic cholecystectomies. Journal of postgraduate medical institute.2006;22(01):66-70.

[9]. Memon W, Khanzada TW, Samad A, Laghari MH. Complications of laparoscopic Cholecystectomy at Isra university Hospital, Hyderabad. Pak J Med Sci.2009;25(1):69-73.

[10]. Mir MA, Malik UY, Wani H, Bali BS. Prevalence, pattern, Sensitivity and resistance to antibiotics of different bacteria isolated from port site infection in low risk patients after elective laparoscopic Cholecystectomy for symptomatic Cholelithiasis at tertiary care hospital of Kashmir. Int wound J.2013;10:110-113.

[11]. Shindholimeth VV, Seenu N, Parshed R , Chaudhury, Kumar R ,Kumar A. Factors influencing wound infection following Laparoscopic Cholecystectomy. Trop Gastroenterology.2003;24:90-2.

[12]. Shah AK,Gambhir RP,Hazra N, Kotoch R . Non Tuberculous mycobacteria in surgical wounds-A rising cause of concern?.Indian J surg.2010;72: 206-10. 